\title{
SIMULATION OF VORTEX STRUCTURE IN SUPERSONIC FREE SHEAR LAYER USING PSE METHOD
}

\author{
XIN GUO AND QIANG WANG \\ China Academy of Aerospace Aerodynamics \\ Beijing 100074, P.R.China University \\ guoxincn@sina.com, qwang327@163.com
}

\begin{abstract}
The method of parabolized stability equations (PSE) are applied in the analysis of nonlinear stability and the simulation of flow structure in supersonic free shear layer. High accuracy numerical techniques including self-similar basic flow, high order differential method, appropriate transformation and decomposition of nonlinear terms are adopted and developed to solve the PSE effectively for free shear layer. The spatial evolving unstable waves which dominate the flow structure are investigated through nonlinear coupling spatial marching methods. The nonlinear interactions between harmonic waves are further analyzed and instantaneous flow field are obtained by adding the harmonic waves into basic flow. Relevant data agree well with that of DNS. The results demonstrate that T-S wave does not keeping growing exponential as the linear evolution, the energy transfer to high order harmonic modes and finally all harmonic modes get saturation due to the nonlinear interaction; Mean flow distortion is produced by the nonlinear interaction between the harmonic and its conjugate harmonic, makes great change to the average flow and increases the thickness of shear layer; PSE methods can well capture the large scale nonlinear flow structure in the supersonic free shear layer such as vortex roll-up, vortex pairing and nonlinear saturation.
\end{abstract}

Keywords: Supersonic free shear layer; parabolized stability equations; nonlinear stability.

\section{Introduction}

The study of supersonic free shear flow is a complicate, unsteady and nonlinear fluid mechanic issue. The study of mixing mechanism in free shear layer has great theoretical and engineer importance, such as to the improving of the mixing intensity in scramjet, and the suppression of the aero-optic phenomena and the radiation of aeroacoustic noise in high speed flows ${ }^{1-3}$. As the flow structure of free shear layers is dominated by instability modes ${ }^{4,5}$, the stability study of disturbance forms the basis of the active and passive control of supersonic free shear layer.

The method of parabolized stability equations (PSE) ${ }^{6}$ is different from the direct numerical simulation (DNS) by which the instantaneous flow quantities or disturbance quantities are solved. The PSE method decomposes the disturbances into multiple modes in Fourier space by Fast Fourier Transform. The assumption of slow-varying in streamwise direction makes PSE solved by spatial marching method. This leads to the highly efficient simulation of flow field. Unlike the linear stability theory (LST) ${ }^{7}$, the parallel hypothesis is not employed and the amplitude of disturbance is not strictly restrained, 
PSE can consider the nonparallel and nonlinear effect ${ }^{8,9}$, and is an effective way in the stability analysis of supersonic free shear layer.

The nonlinear development is one of most important stage in the evolving of disturbances. The nonlinear PSE is adopted in the stability analysis of supersonic free shear layer in this article. High order accuracy method are developed and used to simulate the flow structure for supersonic free shear layer.

\section{Governing Equations}

\subsection{Basic Flow Equations}

Development of basic flow solution for supersonic laminar free shear layer flows begins with the two dimensional compressible boundary layer equations. Similarity solution for shear layer can be found by coordinate transformations $\xi=\rho_{\mathrm{e}} u_{\mathrm{e}} \mu_{\mathrm{e}} x, \eta=\sqrt{\operatorname{Re}} / \rho_{\mathrm{e}} x \int_{0}^{y} \rho d y$. Then, by assuming mixing layer is fully developed and the quantities are only dependent on $\eta$, the basic flow equations are

$$
\begin{aligned}
& \left(\rho \mu f^{\prime \prime}\right)^{\prime}+\frac{1}{2} f \cdot f^{\prime \prime}=0 \\
& \frac{1}{2} c_{p} \cdot f \cdot g^{\prime}+\frac{1}{\operatorname{Pr}} \cdot\left(\kappa \cdot g^{\prime} \cdot \rho\right)+\mathrm{Ec} \cdot \rho \mu \cdot\left(f^{\prime \prime}\right)^{2}=0
\end{aligned},
$$

where the first derivative of $f$ to $\eta$ is $f^{\prime}=u / u_{\mathrm{e}}$, and $g=T / T_{\mathrm{e}} . c_{p}, \mu, \kappa$ and $\rho$ are nondimensional quantities, nondimensionalized with corresponding quantities of free flow; $\operatorname{Pr}=c_{p} \mu / \kappa$, $\mathrm{Ec}=u_{\mathrm{e}}{ }^{2} /\left(c_{p \mathrm{e}} T_{\mathrm{e}}\right)$. The density can be eliminated through nondimensional status equation $\rho=1 / g, \mu$ and $\kappa$ can be expressed as functions of temperature and the ordinary differential equations about $f$ and $g$. For mixing layer, the boundary conditions are

$$
\begin{aligned}
& f^{\prime}(-\infty)=U_{2} / U_{1} \quad f^{\prime}(+\infty)=1 \quad f(0)=0 \\
& g(-\infty)=T_{2} / T_{1} \quad g(+\infty)=1
\end{aligned}
$$

\subsection{Parabolized Stability Equations}

The instantaneous quantities in compressible Navier-Stokes equations and gas equation of state are decomposed into basic quantities and fluctuating quantities. The two dimensional nonlinear disturbance equations are

$$
\Gamma^{l} \frac{\partial \Phi}{\partial t}+A^{l} \frac{\partial \Phi}{\partial x}+B^{l} \frac{\partial \Phi}{\partial y}+D^{l} \Phi+V_{x x}^{l} \frac{\partial^{2} \Phi}{\partial x^{2}}+V_{x y}^{l} \frac{\partial^{2} \Phi}{\partial x \partial y}+V_{y y}^{l} \frac{\partial^{2} \Phi}{\partial y^{2}}=F^{n} .
$$

The disturbance vector $\Phi=[u, v, \rho, T, p]^{\mathrm{T}}$, and $F^{n}$ is the nonlinear term. According to the fast Fourier transformation and PSE theory ${ }^{6}$, the fluctuating components are modeled with the general form of traveling waves, i.e.

$$
\Phi=\sum_{m=-\infty}^{+\infty} A\left(x_{0}\right)_{m} \cdot \psi_{m} \chi_{m},
$$


where $\psi_{m}$ and $\chi_{m}$ are slow-varying shape functions and wave-like functions of mode $m$

$$
\psi_{m}(x, y)=[\tilde{u}, \tilde{v}, \tilde{\rho}, \tilde{T}, \tilde{p}]_{m}^{\mathrm{T}}, \chi_{m}=\exp \left[\mathrm{i} \cdot\left(\int_{x_{0}}^{x} \alpha_{m}(x) d x-m \omega t\right)\right] .
$$

$A\left(x_{0}\right)_{m}$ is the initial amplitude, $m \omega$ and $\alpha_{m}$ are frequency and wave number for mode $m$. Due to the slow-varying property ${ }^{8}$, the second order derivatives and products of first order derivatives for shape functions in streamwise direction are negligible, so that

$$
\frac{\partial^{2} \Phi}{\partial x^{2}}=\sum_{m=-\infty}^{+\infty}\left[-\alpha_{m}^{2} \psi_{m} \cdot \chi_{m}+2 \mathrm{i} \cdot \alpha_{m} \frac{\partial \psi_{m}}{\partial x} \cdot \chi_{m}+\mathrm{i} \cdot \frac{d \alpha_{m}}{d x} \psi_{m} \chi_{m}\right]
$$

Substituting Eqs. (3) (5) into Eq. (2), the nonlinear parabolized stability equations (NPSE) are further obtained

$$
\hat{D}_{m} \psi_{m}+\hat{A}_{m} \frac{\partial \psi_{m}}{\partial x}+\hat{B}_{m} \frac{\partial \psi_{m}}{\partial y}+V_{y y}^{l} \frac{\partial^{2} \psi_{m}}{\partial y^{2}}=\frac{\left[F^{n}\right]_{m}}{\chi_{m}} .
$$

\section{Numerical Methods}

\subsection{Coordinate Transformation and Discretization}

The conflicting needs to offer high resolution of the flow in the shear region while still requiring the computational domain to extend far into each free stream demand the use of grid stretching. The mapping used here

$$
y=\left(L_{y} / 2\right) \cdot \sinh \left(b_{y} \eta\right) / \sinh \left(b_{y}\right) .
$$

The mapping coordinate $\eta \in[-1,1]$, and physical domain $y \in\left[-L_{y} / 2, L_{y} / 2\right] . L_{y}$ and $b_{y}$ are control parameter.

The forth order central differential method are used for discretization in normal direction.

$$
\begin{aligned}
& \frac{\partial \psi_{i, j}}{\partial \eta}=\frac{-\psi_{i, j+2}+8 \psi_{i, j+1}-8 \psi_{i, j-1}+\psi_{i, j-2}}{12 \Delta \eta} \\
& \frac{\partial^{2} \psi_{i, j}}{\partial \eta^{2}}=\frac{-\psi_{i, j+2}+16 \psi_{i, j+1}-30 \psi_{i, j}+16 \psi_{i, j-1}-\psi_{i, j-2}}{12 \Delta \eta^{2}}
\end{aligned} .
$$

While the formulas applied at the point adjacent to boundary are ( $j=2$ for example).

$$
\begin{aligned}
& \frac{\partial \psi_{2}}{\partial \eta}=\frac{\psi_{i, 5}-6 \psi_{i, 4}+18 \psi_{i, 3}-10 \psi_{i, 2}-3 \psi_{i, 1}}{12 \Delta \eta} \\
& \frac{\partial^{2} \psi_{i, 2}}{\partial \eta^{2}}=\frac{\psi_{i, 6}-6 \psi_{i, 5}+14 \psi_{i, 4}-4 \psi_{i, 3}-15 \psi_{i, 2}+10 \psi_{i, 1}}{24 \Delta \eta^{2}}
\end{aligned} .
$$


The first order backward differential formula $\partial \psi_{i, j} / \partial x=\left(\psi_{i, j}-\psi_{i-1, j}\right) / \Delta x$ is chosen for streamwise derivative, and the truncation error is $\Delta x^{2} \partial^{2} \psi / \partial x$ where PSE slow-varying assumption is appropriate.

The disturbance is zero at the infinite far field. However, the finite difference method could not contain the infinite domain usually. Since the viscous effect is negligible at far field, the governing equations at the boundaries are derived from inviscid stability equations $^{10,11}$, can be written as following form

$$
y= \pm y_{\max }, \frac{\partial \psi}{\partial y}=\mp q \cdot \psi, q=\sqrt{\bar{\rho} /\left(1 / \bar{\rho}-M_{1}^{2}(\bar{u}-\omega / \alpha)^{2} / \gamma\right)} \cdot \alpha_{m} .
$$

The first order derivatives at boundaries are substituted by forth order partial differential formula

$$
\begin{aligned}
& \frac{\partial \psi}{\partial \eta}=\frac{1}{12 \Delta \eta}\left(-3 \psi_{5}+16 \psi_{4}-36 \psi_{3}+48 \psi_{2}-25 \psi_{1}\right) \\
& \frac{\partial \psi}{\partial \eta}=\frac{1}{12 \Delta \eta}\left(25 \psi_{N}-48 \psi_{N-1}+36 \psi_{N-2}-16 \psi_{N-3}+3 \psi_{N-4}\right)
\end{aligned}
$$

Finally, the matrix equation are obtained

$$
\left[L_{m}^{i+1}+M_{m}^{i+1} / \Delta x\right] \cdot \psi_{m}^{i+1}=M_{m}^{i+1} / \Delta x \cdot \psi_{m}^{i}+F_{m}^{i+1} / A_{m}^{i+1} .
$$

\subsection{Analysis of Nonlinear Terms}

We perform the calculation of nonlinear interaction in a different way from Ref.10, in which, the calculation procedure starts with an inverse fast Fourier transform of the flow variables from Fourier to physical space to produce disturbance information at discrete space and time positions. The nonlinear terms are then evaluated in real space to produce the total nonlinear forcing vector $F^{n}$. Finally, a forward FFT is used to determine $\left(F^{n}\right)_{m}$, the forcing contribution to the different stability modes in Fourier space. Here, we derived the nonlinear terms expression in an analytical way.

The symmetry condition for harmonic waves in frequency field is applied to reduce the computational cost. The harmonics with negative frequency are expressed as

$$
A_{m}=A_{-m}, \psi_{-m}=\left[\tilde{u}_{-m}, \tilde{v}_{-m}, \tilde{\rho}_{-m}, \tilde{T}_{-m}, \tilde{p}_{-m}\right]^{\mathrm{T}}=\left[\tilde{u}_{m}^{+}, \tilde{v}_{m}^{+}, \tilde{\rho}_{m}^{+}, \tilde{T}_{m}^{+}, \tilde{p}_{m}^{+}\right]^{\mathrm{T}},
$$

where the superscript ' + ' denotes to the conjugate, A is the amplitude. It is implied that the harmonic waves with $m<0$ do not need to be evaluated. Phase lock condition is also applied to determine the wave numbers of high order harmonic waves, i.e, $\left(\alpha_{m}\right)_{\mathrm{r}}=m \cdot\left(\alpha_{m}\right)_{\mathrm{r}}$

High order terms in $F^{n}$ are neglected, so that the nonlinear contribution to any harmonic wave $m$ can be expressed as the sum of the interactions between any two harmonics $m_{1}$ and $m_{2}$ if $m_{1}+m_{2}=m$, and can be written as following

$$
F^{n}(m)=\sum_{m_{1}=-\infty}^{+\infty} N\left(m_{1}, m_{2}\right) \cdot \chi_{m_{1}} \chi_{m_{2}}, \text { where } \mathrm{m} 2=\mathrm{m}-\mathrm{m} 1 .
$$


$N\left(m_{1}, m_{2}\right)$ is the nonlinear interaction factor of shape function ${ }^{12}$. Substituting $N$ into Eq. (6), and eliminating the wave function, following nonlinear term are obtained

$$
\frac{F^{n}(m)}{\chi_{m}}=\sum_{m_{1}=-\infty}^{+\infty} N\left(m_{1}, m_{2}\right) \frac{A_{m_{1}} A_{m_{2}}}{A_{m}} .
$$

where $A$ is the amplitude of harmonic wave.

\subsection{Spatial Marching Procedure}

Streamwise change of $\Phi$ in Eq. (2) can be absorbed into either the shape function $\psi_{m}(x, y)$ or the complex streamwise wave number $\alpha_{m}(x)$, where the latter defines the value of the amplitude function. This ambiguity must be resolved through the introduction of an additional constraint. The constraint on the streamwise growth of $\psi_{m}(x, y)$ is usually imposed, which is also called normalization condition. The present investigation adopt the following formula to constrain the streamwise change of $\psi_{m}(x, y)$

$$
\int_{-y_{\max }}^{y_{\max }}(\tilde{v})_{m}^{+}\left(\tilde{v}_{x}\right)_{m} d y=0
$$

The predict-correct method is employed in the spatial marching procedure. The correcting step of $\alpha$ for each mode is following the normalization condition

$$
\Delta \alpha=-\mathrm{i} \cdot \int_{-y_{\max }}^{y_{\max }} \tilde{v}_{i}^{+}\left(\tilde{v}_{i}-\tilde{v}_{i-1}\right) d y /\left(\Delta x \cdot \int_{-y_{\max }}^{y_{\max }} \tilde{v}_{i}^{+} \tilde{v}_{i} d y\right) .
$$

The nonlinear spatial marching processes usually are

(a) predicting the disturbance $\left(\alpha_{m}, A_{m}\right.$ and $\left.\psi_{m}\right)$ at position $i$ by the solution at $i-1$

(b) solving the parabolized stability equation to get new $\psi_{m}$

(c) using normalization condition to correct the predicted $\alpha_{m}$ and $A_{m}$

(d) repeating (b) (d) until $\Delta \alpha_{m}$ is sufficient small

(e) repeating (b) (e) to get all harmonic waves

(f) repeating (b) (f) until all harmonics convergent

(g) moving downstream for the next station $i+1$.

\subsection{Initial Conditions}

Initial disturbances are needed before marching forward. For linear PSE, the results of LST are usually used as the initial condition. LST equation can be derived from PSE by ignoring the streamwise derivatives and vertical velocity component, is in form of

$$
L_{m} \cdot \psi_{m}=0
$$

It forms the eigenvalue problem of frequency and wave number of disturbance, and the Newton iteration is used to solve it. 
For NPSE, all harmonics should be solving simultaneously in each marching step because of the interaction with each other. Providing that the initial amplitude is sufficient small, the LPSE results are suitable for the linear unstable harmonic waves. However, for high order harmonic waves which is usually linear stable, a parallel version of nonlinear stability equations without streamwise derivatives and vertical velocity component are solved to get the initial conditions, which is in form of

$$
L_{m} \cdot\left(A_{m} \psi_{m}\right)=F_{m}^{n}
$$

The real part of unknown parameter $\alpha_{m}$ in coefficient matrix is given by phase lock condition, and the imaginary part is given by nonzero small value to avoid the singularity of the equation. $F_{m}^{n}$ in this equation is the nonlinear forcing vector produced by interactions between low order harmonic waves. When $A_{m} \psi_{m}$ is available, the $A_{m}$ and $\psi_{m}$ can obtained from unitary condition (the max value in $\psi_{m}$ is set to unit one).

\section{Results and Analysis}

\subsection{Basic Flow and Initial Disturbances}

The forth order Runge-Kutta method and shooting method are employed to solve the basic flow equation. The integration starts from $y=0$, and ends at $y= \pm y_{\max } \cdot f^{\prime}, f^{\prime \prime}, g$ and $g^{\prime}$ at $y=0$ are predicted and revised. Repeating of the integration process terminate when the boundary conditions are satisfied.

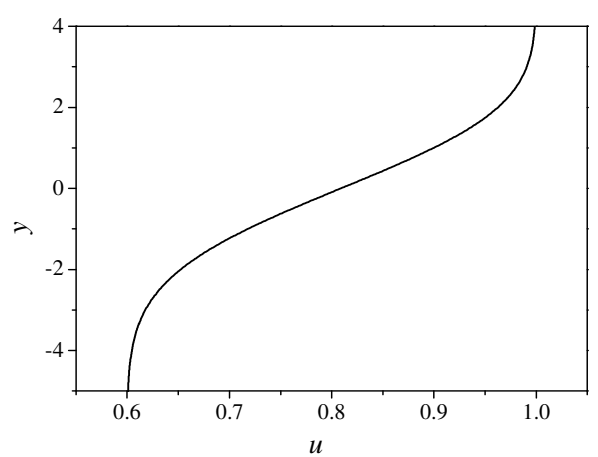

Fig. 1. Streamwise velocity distribution

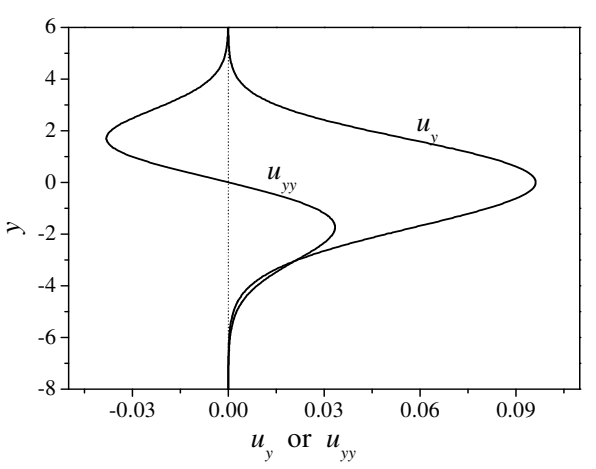

Fig. 2. Derivatives of streamwise velocity

The distribution of streamwise velocity and its vertical derivatives of laminar flow are given in Fig. 1 and Fig. 2 respectively. The free flow conditions are: $M_{1}=2.5, M_{2}=1.5$, $\rho_{1}=\rho_{2}, \operatorname{Pr}=1.0$. The coordinate $y=y^{*} / \delta_{x}$, the vortex thickness $\delta_{\omega}=4.16 \delta_{x}$. As shown in Fig. 2, velocity profile gets an inflectional point $u_{y y}=0$ near the area $y=0$, which demonstrate the inviscid unstable property ${ }^{13}$ of mixing layer. 


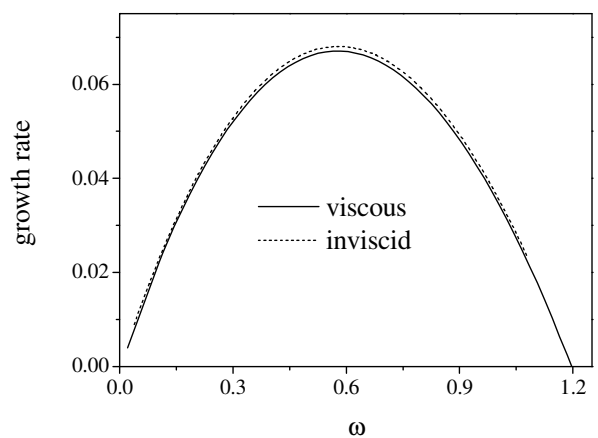

Fig. 3. Growth rate vs. frequency

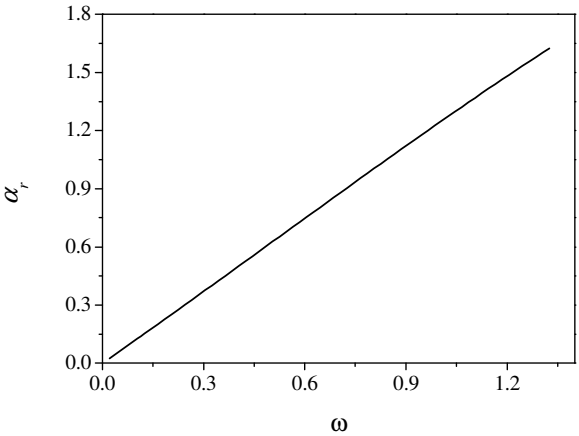

Fig. 4. Wavenumber vs. frequency

The Reynolds number at the inlet is set to 5000. The LST equation is solved for stability analysis. Fig. 3 shows the spatial growth rate distribution with the frequency. The frequencies, wave number and growth rate of the most amplified disturbance are respectively $\omega=0.5781, \alpha_{\mathrm{r}}=0.7194$ and $-\alpha_{\mathrm{i}}=-0.06806$. The growth rate of inviscid stability results are also given in the figure with dash line, which is a little smaller than the viscous ones, and demonstrate that viscosity has the stabilizing effect. The wave number shown in Fig. 4 is linear change with the frequency, verifying the phase lock condition.

\subsection{Nonlinear Evolution}

\section{Case 1 Nonlinear evolution of fundamental wave}

The evolution of 17 harmonics ( $m=-8 \sim 8$ ) are considered in case 1. Two frequency modes, the fundamental $(m=1)$ and first harmonic $(m=2)$, are specified at the inlet using their LPSE solutions. The fundamental mode has the frequency of most amplified disturbances. The High order modes $(m>3)$ and mean flow distortion $(m=0)$ are calculated by Eq. (10). The detail parameters of all modes are listed in table 1 .

Table 1 Initial disturbances at the inflow

\begin{tabular}{c|c|c|c|c}
\hline$m$ & $\omega$ & $A$ & $A_{0}$ & Produced by \\
\hline 1 & 0.5781 & $(0.7194,-0.06701)$ & $5 \times 10^{-4}$ & linear unstable \\
\hline 2 & 1.1562 & $(1.4301,-0.00809)$ & $1 \times 10^{-4}$ & linear unstable \\
\hline 0 & 0.0000 & $(0.0000,-0.01000)$ & $2.8438 \times 10^{-5}$ & $(1,-1) ;(2,-2)$ \\
\hline 3 & 1.7343 & $(2.1582,-0.01000)$ & $8.9036 \times 10^{-7}$ & $(1,2) ;(2,1)$ \\
\hline 4 & 2.3124 & $(2.8776,-0.01000)$ & $4.4019 \times 10^{-8}$ & $(1,3) ;(2,2) ;(3,1)$ \\
\hline 5 & 2.8905 & $(3.5970,-0.01000)$ & $3.6814 \times 10^{-9}$ & $(1,4) ;(2,2) ;(3,1)$ \\
\hline 6 & 3.4686 & $(4.3164,-0.01000)$ & $2.3467 \times 10^{-10}$ & $(1,5) ;(2,4) ;(3,3) ;(4,2) ;(5,1)$ \\
\hline 7 & 4.0467 & $(5.0358,-0.01000)$ & $3.5276 \times 10^{-11}$ & $(1,6) ;(2,5) ;(3,4) ;(4,3) ;(5,2)(1,6)$ \\
\hline 8 & 4.6248 & $(5.7552,-0.01000)$ & $8.4775 \times 10^{-14}$ & $(1,7) ;(2,6) ;(3,5) ;(4,4) ;(5,3) ;(6,2) ;(1,7)$ \\
\hline
\end{tabular}


The evolution of fundamental disturbance and second harmonic are given in Fig. 5, the growth rates agree well with the DNS results of Ref. 10, especially before the disturbance getting saturation. The NPSE finishes the simulation in less than one hour, and the cost is much smaller than that of DNS. Due to the nonlinear interaction, fundamental wave does not keep growing exponentially as the linear development (LPSE, dash line) as shown in the figure. When the amplitudes get large enough, the disturbance of fundamental wave get into stage of nonlinear evolution and the growth rate decrease gradually. Finally, harmonics become saturation.

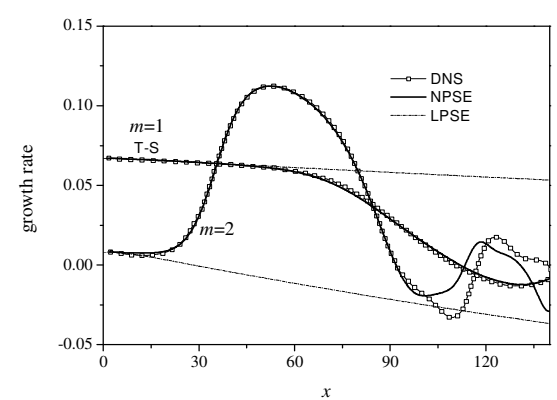

Fig. 5. Growth evolution of modes $m=1$ and $m=2$

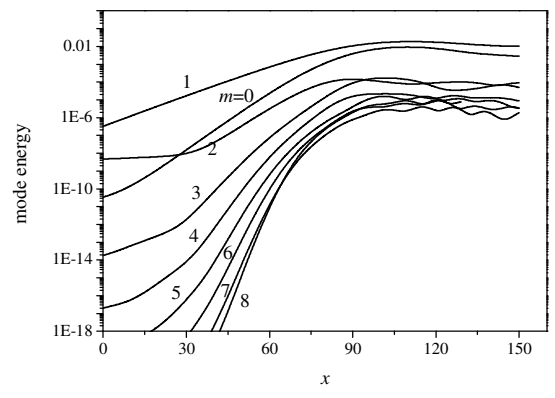

Fig. 6. Energy evolution of harmonic modes

Fig. 6 shows the energy evolution of all harmonics. It should be noted that high order harmonics enter the strong nonlinear increase stage initially. The energy transfer to high order harmonics, at about $x=100$, all harmonics get saturation. It is conclude that, since the infinite small disturbances appearing in the flow, the linear effect makes the unstable disturbances developed to a high amplitude level; and the nonlinear effect redistribute energy in the Fourier domain, and transfer the energy from average flow and low frequency modes to high order modes.

Nonlinear interactions between modes and their conjugate ones wave result in contributions at zero frequency mode, which is called mean flow distortion (MFD). The energy of MFD gets up to the level fundamental wave as shown in Fig. 6, and makes great change of average flow. Fig. 7 and Fig. 8 give velocity of the average flow and mean flow distortion at different locations. The vertical coordinate is similarity coordinate in which similar basic flow profiles at different location are the same. The similarity does not exist in the downstream when mean flow distortion is large enough. The profiles imply that mean flow distortion increases the thickness of shear layer.

Since the shape functions, wave numbers and growth rates are available by spatial marching procedure, the instantaneous flow field is obtained by adding all harmonic waves into basic flow, and is used for analysis of the flow structure. The instantaneous flow field is performed in Figs. 9 12, including contour lines of streamwise velocity, temperature, pressure and vortex respectively. It should be noted that the method of 


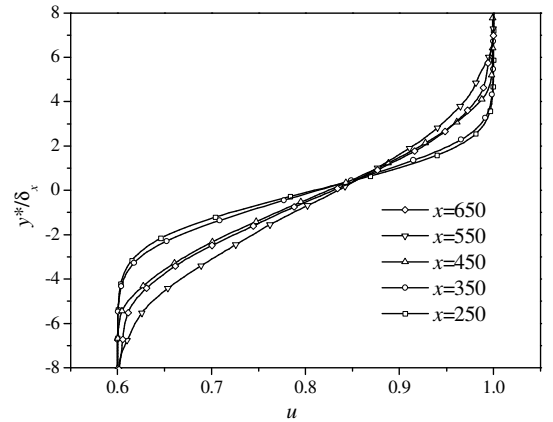

Fig. 7. Streamwise velocity of average flow

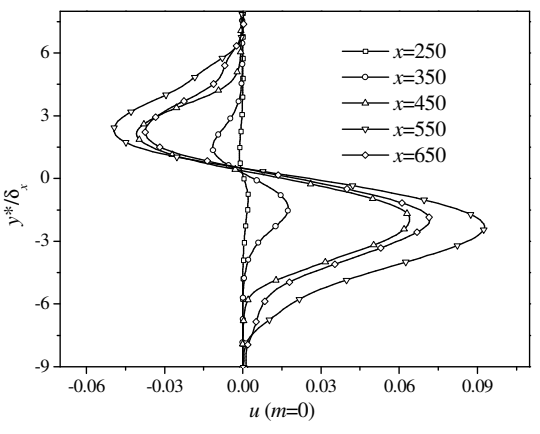

Fig. 8. Streamwise velocity of mean flow distortion

NPSE can fast and effectively capture the nonlinear large scale vortex structure, which makes NPSE an effective tool in the simulation of shear flow. Fig. 12 clearly demonstrates the formation, development and saturation of vortex. At about $x=60$, the fundamental mode which has the highest energy level, enter into the stage of nonlinear development. As shown in Fig.5, the growth rate gradually separate from the linear stability results. With the fast nonlinear growth of high order harmonics, the vortex is gradually rolled-up and enlarged. The size vortex get largest at the region $x=110 \sim 120$, and finally saturate.

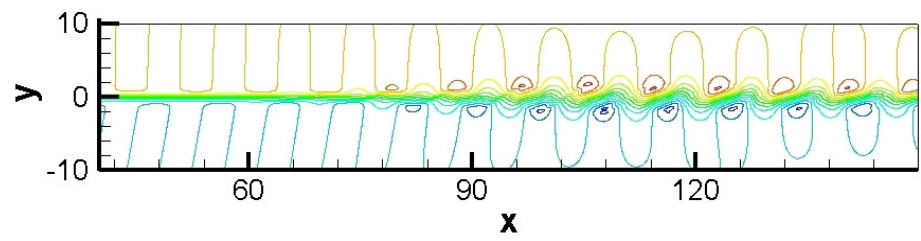

Fig. 9. Instantaneous velocity $(\mathrm{u}=0.5 \sim 1.1, \Delta \mathrm{u}=0.05)$

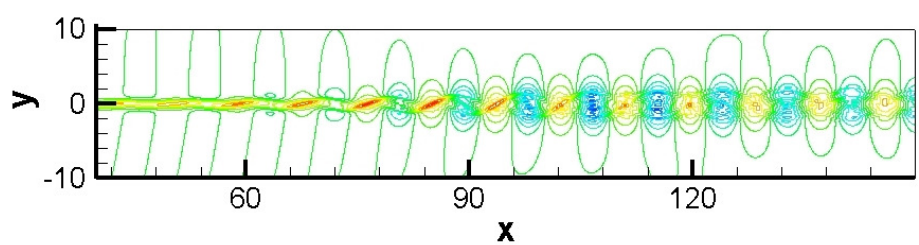

Fig. 10. Instantaneous temperature $(\mathrm{T}=0.94 \sim 1.07, \Delta \mathrm{T}=0.01)$

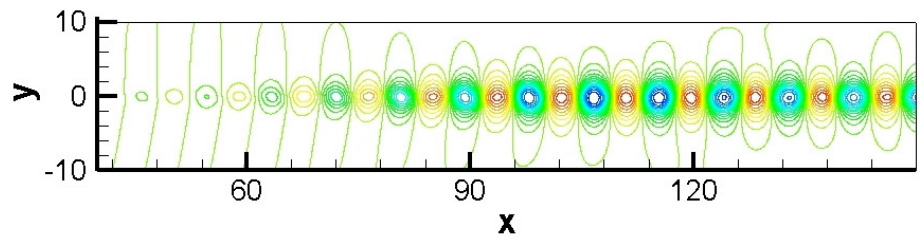

Fig. 11. Instantaneous pressure $(\mathrm{p}=0.72 \sim 1.16, \Delta \mathrm{p}=0.02)$ 


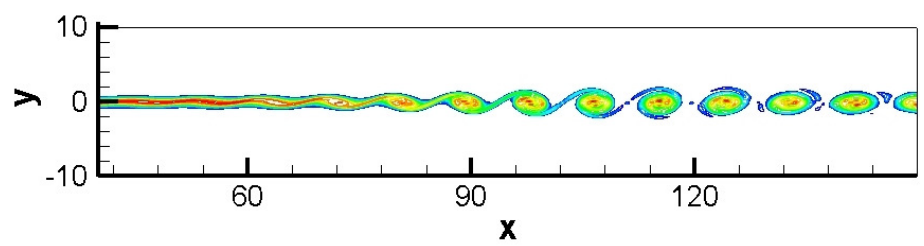

Fig. 12. Instantaneous vortex $(\omega \mathrm{z}=0.06 \sim 0.36, \Delta \omega \mathrm{z}=0.03)$

\section{Case 2 Nonlinear evolution of fundamental and subharmonic wave}

The results in case 1 demonstrate that PSE does not eliminate the importance physics of vortex rolling up. In case 2, NPSE is used to investigate the nature of vortex pairing. The evolution of 29 harmonics ( $m=-14 \sim 14)$ are considered. The main disturbances at the inlet are fundamental wave and its subharmonic wave, which are labeled as $m=2$ and $m=1$ respectively. The detail parameters are given in table 2 . The high order harmonics and mean flow distortion are also calculated in the same way as case 1 .

Table 2 main disturbances at Inlet for case 2

\begin{tabular}{c|c|c|c}
\hline$m$ & $\omega$ & $A$ & $A_{0}$ \\
\hline 1 & 0.4 & $(0.4955,-0.06103)$ & $1 \times 10^{-3}$ \\
\hline 2 & 0.8 & $(0.9977,-.0 .05798)$ & $4 \times 10^{-3}$ \\
\hline
\end{tabular}

Harmonic energy evolutions are shown in Fig. 13. Different from case 1, the fundamental wave $(m=2)$ and its harmonics wave $(m=1)$ dominate the flow field. High order harmonics $(m=3 \sim 14)$ get to high energy level through strong nonlinear growth, and finally get saturation. The instantaneous vortex contours floods are performed in Fig. 14. At the region $x=30 \sim 70$, the fundamental mode get saturation, and the vortex roll up; the subharmonic mode grow fast and surpass the fundamental wave at the position $x=70$, and a pair of vortex merge into a new one in large size during $x=70 \sim 100$. The distances of the center of the vortex became twice of the wave length of the fundamental wave.

\section{Conclusions}

The nonlinear parabolized stability equations are employed in the simulation of supersonic free shear layer. The similar boundary layer equations are solved to get the laminar flow. The linear unstable waves with finite small amplitudes are introduced at the inflow. The coupling evolutions of unstable waves and its harmonic waves are investigated by solving NPSE. It is concluded that

(a) The spatial marching procedure of effective harmonic modes in NPSE makes NPSE an effective method in the simulation of the nonlinear evolution of disturbances. 


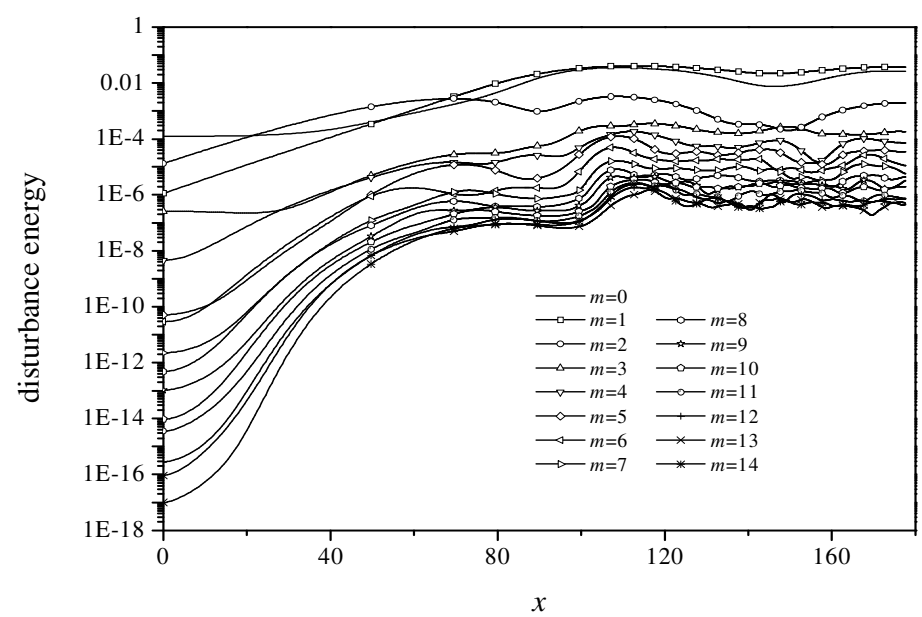

Fig.13. Evolution of harmonic energy

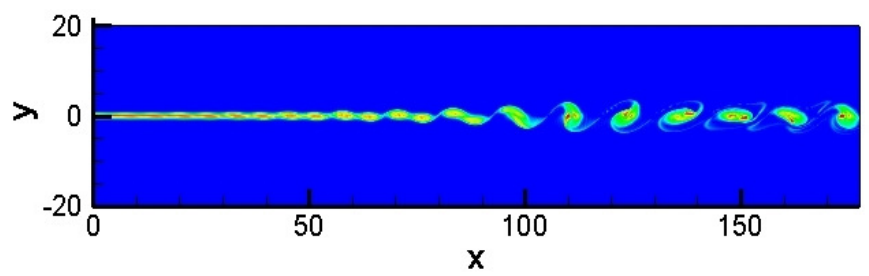

Fig. 14. Instantaneous vortex $(\omega \mathrm{z}=0.03 \sim 0.42, \Delta \omega \mathrm{z}=0.03)$

(b) Nonlinear interaction makes the energy transfer to high order harmonic waves. High order harmonic waves grow rapidly to high level of amplitudes, and get saturation finally.

(c) Nonlinear interactions between harmonic modes and its conjugate ones produce the mean flow distortion, makes great change in average flow, and increasing the thickness of mixing layer.

(d) NPSE can well capture the large scale structure in supersonic shear layer, and correctly simulate development of the nonlinear vortex structure.

\section{References}

1. C. Bodony and C. Bailly, AIAA J. 38, 2210 (2000).

2. M. L. Shelah, L. C. Rodney and F. C. Randy, Proc. SPIE, 1326, 190 (1990).

3. X. Zhang, AIAA J. 33, 8 (1995).

4. L. S. Huang and C. M. Ho, J. Fluid Mech. 210, 475 (1990)

5. L. C. Cheung and S. K. Lele, Standford University Phd. (2007).

6. T. Herbert, Annu. Rev. Fluid Mech. 29, 245 (1997). 
7. N. D. Sandham and W. C. Reynolds, AIAA J. 28, 618 (1990).

8. F. P. Bertolotti, T. Herbert P. R. and Spalart, J. Fluid Mech. 242, 441 (1992).

9. C. L. Chang and M. R. Malik, ICASE Report No.93-70 (1993).

10. M. J. Day, N. N. Mansour and W. C. Reynolds, J. Fluid Mech. 446, 375 (2001).

11. L. C. Cheung and S. K. Lele, AIAA Paper, 2004-0363 (2004).

12. X. Guo and D. B. Tang, Chinese J. Aero. 23, 283 (2010).

13. T. L. Jackson and C. E. Grosch, ICASE Report, No.89-32 (1989). 